\title{
Study of Novel AuCu-Pt Trimetallic Multiply Twinned Nanoparticles with High Index Surfaces
}

\author{
Subarna Khanal, Nabraj Bhattarai, J. Jesus Velazquez-Salazar, and Miguel Jose-Yacaman \\ Department of Physics and Astronomy University of Texas at San Antonio, One UTSA Circle, San \\ Antonio, Texas, 78249
}

The synthesis of noble metals nanoparticles with controlled atomic distributions have attracted significant interest due to their size-tunable properties depending up on the variation of composition and structure. Recently, various researchers have been focused on the fabrication of multimetallic nanoparticle catalysts due to their tunable properties implemented in diverse potential applications. The addition of a third metal in to the nanoparticle catalyst is expected to produce a combination of effects such as modification of the electronic structure, change in the d-band center, reduction of the lattice distance and enhances the overall electronic charge shift. The control morphology of the nanostructures can provide a great opportunity to stimulate their possible applications such as catalytic, electronic, sensing, and optical properties and to enhance their reactivity, selectivity and stability. [1-3] AuCu-Pt trimetallic nanocrystals with multiply twinned structures, possessing the penta-twinned morphology with a high high-index surfaces of Pt atoms on the (111) surfaces are expected to be the most active catalysts for their many applications in fuel cells.

The morphology of the nanoparticles was characterized by transmission electron microscope (TEM) and high resolution TEM operated at $200 \mathrm{kV}$ with a $0.19 \mathrm{~nm}$ point resolution. The scanning transmission electron microscopy (STEM) images were recorded in a probe Cs-corrected Microscope operated at $200 \mathrm{kV}$. High angle annular dark field (HAADF) STEM images were obtained with a convergence angle of $26 \mathrm{mrad}$ and the collection semi-angles from 50 to $180 \mathrm{mrad}$. These variations in semi-angles satisfy the conditions set forth for the detectors to eliminate contributions from unscattered and low-angle scattered electron beams. The probe size used was about $0.09 \mathrm{~nm}$ with the probe current of $22 \mathrm{pA}$. In addition, bright field (BF) STEM images were recorded by using a collection semi-angle of $11 \mathrm{mrad}$. Electron dispersive X-ray spectroscopy (EDS) spectra were obtained using a probe size of $0.13 \mathrm{~nm}$ with the probe current $86 \mathrm{pA}$.

In this work, we present the synthesis of $\mathrm{AuCu}-\mathrm{Pt}$ trimetallic nanoparticles with penta-twinned structure, consisting of a Pt monolayer shell on the top of $\mathrm{AuCu}$ core by one-pot colloidal synthesis method. The atomic resolution Cs-corrected STEM in combination with high resolution spectral and chemical analysis has been allowed us to see the Pt layers are overgrowth on as prepared AuCu core by Frankvan der Merwe layer-by-layer and Stranski-Krastanov island-on-wetting-layer growth modes, the atomic structure of $\mathrm{AuCu} / \mathrm{Pt}$ trimetallic nanoparticles and the chemical composition of different elements were found from the EDS analysis. The obtained nanostructure carries significant importance due to the highly reactive geometry obtained and observed by the atomic resolution microscopy. The presence of significant amount of copper in the structure was shown to make the nanostructures more cost effective and at the same time the presence of Pt atoms decorated surfaces with high index surfaces $\{321\}$ and $\{211\}$ makes them catalytically more active. This work is a step closer to produce optimum catalysts in which the active phase forms only surface monolayers.

\section{References}

[1] N. Toshima et al., Catalysis Today, 2007. 122, 239.

[2] S. Khanal et al., Nanoscale, 2013 
[3] C. R. Ghosh and S. Paria, Chemical Reviews, 2012, 112, 2373.

[4] Acknowledgments: The authors would like to acknowledge NSF grants DMR-1103730, "Alloys at the Nanoscale: The Case of Nanoparticles Second Phase”, PREM: NSF PREM Grant \# DMR 0934218, and Welch Foundation (grant No. AX-1615).
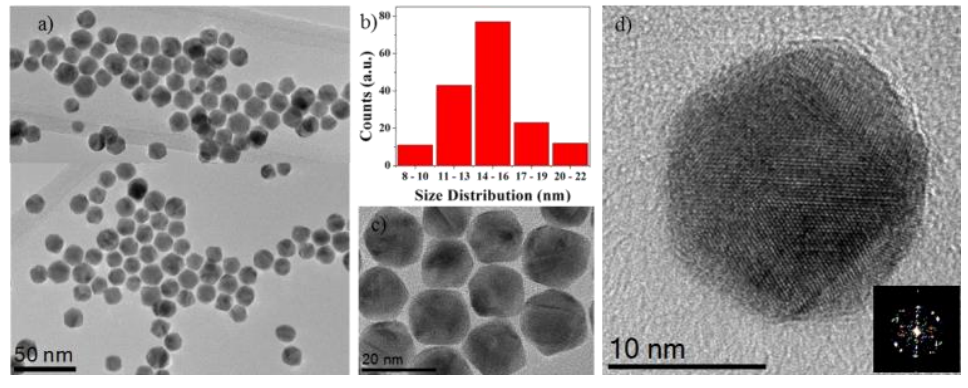

Figure 1. (a) TEM image of AuCu-Pt trimetallic nanoparticles. (b) Histogram shows the particle size ranging from $15.0 \pm 1.0 \mathrm{~nm}$. (b) and (c) HRTEM images of the multiple twinned structure nanoparticles which is oriented along the $\{001\}$ zone axis.
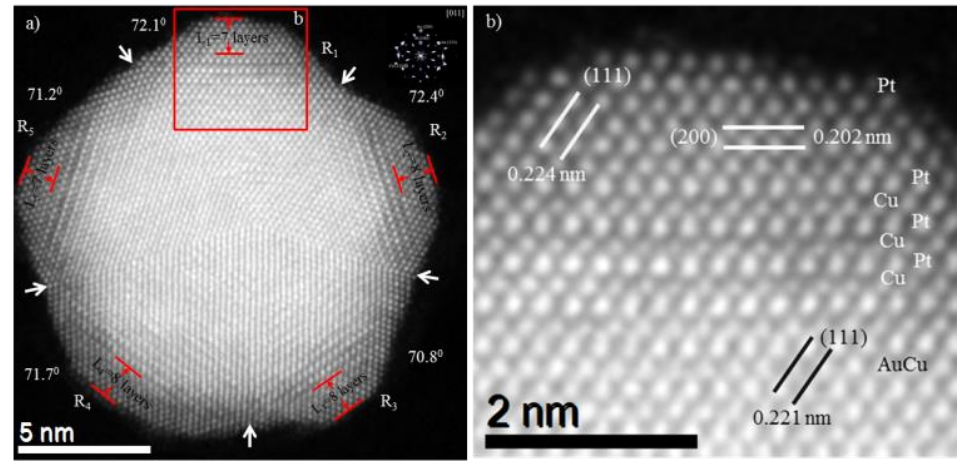

Figure 2. (a) HAADF-STEM image of a penta-twinned AuCu-Pt core-shell nanoparticle. Each tetrahedron (R1, R2, R3, R4, and R5) is identified by white arrow and the angles measured between the $\{111\}$ twinning plane are $72.1^{\circ}, 72.4^{\circ}, 70.8^{\circ}, 71.7^{\circ}$, and $71.2^{\circ}$ as indicated. (b) Close-up of the image as indicated by the square showing the crystalline planes and different contrasts of $\mathrm{Pt}, \mathrm{Cu}$ and $\mathrm{Au}$ lattices are readily observed.
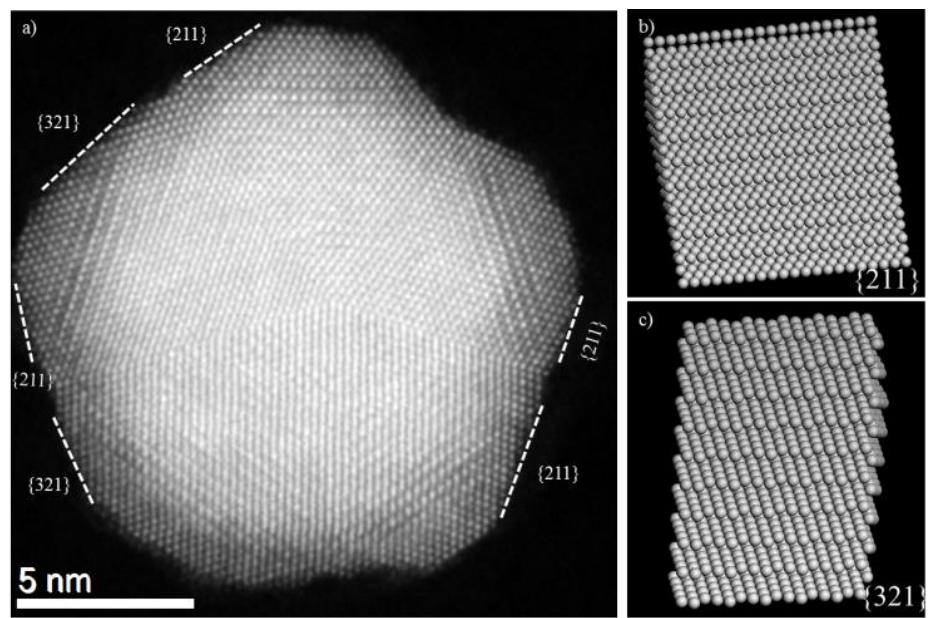

Figure 3. (a) Shows HAADF image of $16 \mathrm{~nm}$ penta-twinned nanoparticles on a [011] zone axis with high index exposed facets, (b and c) Atomistic model for high-index facets $\{211\}$ and $\{321\}$ with steps and terraces. 